\title{
GENERALIZED WEIERSTRASS KERNELS ON THE INTERSECTION OF TWO COMPLEX HYPERSURFACES
}

\author{
FRANCO FERRARI \\ Center for Theoretical Physics, Laboratory for Nuclear Science and \\ Department of Physics, Massachusetts Institute of Technology \\ Cambridge, Massachusetts 02139, USA
}

Physics Department, University of Szczecin, Wielkopolska 15, 70-451 Szczecin, Poland ${ }^{1}$. Physics Department, University of Trento and INFN, Gruppo Coll. di Trento, 38050 Povo, Italy.

\begin{abstract}
On plane algebraic curves the so-called Weierstrass kernel plays the same role of the Cauchy kernel on the complex plane. A straightforward prescription to construct the Weierstrass kernel is known since one century. How can it be extended to the case of more general curves obtained from the intersection of hypersurfaces in a $n$ dimensional complex space? This problem is solved in this work in the case $n=3$. As an application, the correlation functions of bosonic string theories are constructed on a canonical curve of genus four.
\end{abstract}

December 2000

1 E-mail: fferrari@univ.szczecin.pl 


\section{INTRODUCTION}

Algebraic curves and $n$-sheeted branched covers on the projective line $\mathbf{P}^{1}$ provide an explicit representation of abstract Riemann surfaces. Besides being an active field of research in mathematics [1]-[6], since more than a decade they have been also successfully applied in several different topics of theoretical and mathematical physics [7] -[11].

All the above physical applications involve only plane affine algebraic curves. Moreover, most of the works are further restricted to the particular case of hyperelliptic curves. The reason is that the latter are very well known in mathematical literature. An invaluable source of hyperelliptic formulas can be found for instance in the original Weierstrass lecture notes [12]. As an example, we give the prescription of Weierstrass for differentials of the second kind. Already in the case of the so-called $Z_{n}$ symmetric curves, which are the most straightforward generalization of hyperelliptic curves, superstring calculations like those of [7] are impossible, since it has not yet been understood how to construct sections with half-integer spins or chiral determinants [13]-[14] of free fermions with given spin structures. An exception is provided by more exotic fermions with $1 / n$ characteristics, which have been solved in [15]. Also neglecting fermions and sticking to bosonic string theory, the computation of the partition function remains a very complicated task on non-hyperelliptic algebraic curves.

The explicitness of algebraic curves is indeed of great advantage in understanding the physical aspects of theories like superstrings whenever Riemann surfaces are involved, but, on the other side, it is also the main responsible of the above mentioned difficulties. Using 
different approaches, like for instance theta functions, the problems are somewhat hidden, but they reappear in other forms, in particular when one needs to explore a limited portion of the moduli space of Riemann surfaces such as that spanned by $Z_{n}$ symmetric curves.

A considerable effort to understand conformal field theories on plane algebraic curves and to find new applications of non-hyperelliptic curves has been made by the author over the past ten years, partly in collaboration with J. Sobczyk and W. Urbanik. In particular, the applications of branched covers of $\mathbf{P}^{1}$ have been investigated, in which the curves are projected on the complex projective line. Every compact Riemann surface can be represented in this way. Branched coverings are also closely related to affine curves in the two dimensional complex plane $\mathbf{C}^{2}$. The bosonic string theories on a general nonhyperelliptic algebraic curve of genus three have been treated in refs. [16]-[17], computing the correlation functions of the theory and its partition function, the latter up to a theta constant. Moreover, the partition function has been exactly derived on $Z_{3}$ symmetric curves. in [17]. Even in this case, where the chiral determinants are well known [18], the calculation of the partition function is not simple. Indeed, one has still to use the Rauch's variational method [19] combined with the Beilinson-Manin formula [20] in order to determine the explicit form of the period matrix. The same variational approach of Rauch has been exploited in [21] to rederive in an elegant way the Thomae formulae of [18] on $Z_{n}$ symmetric curves.

Despite the difficulties of evaluating the partition functions of conformal field theories, algebraic curves become very convenient in the construction of meromorphic tensors with poles and zeros of given order at given points, like for example the correlation functions of the free fields appearing in the bosonic string action [13]. On general plane algebraic curves, 
this problem has been solved for the $b-c$ systems in [22]-[23]. The more complicated case of scalar fields has been treated recently in [24]. The two and four-point amplitudes of the bosonic $\beta-\gamma$ systems have instead been derived in [25]. Other useful formulas can be found in $[26]$.

Plane algebraic curves are described by complex coordinates which, depending on the interpretation, can be multivalued functions on the complex plane $\mathbf{C}$ or on the complex sphere $\mathbf{P}^{1}$. They change their branches at the branch points according to certain monodromy properties. For this reason, it was thought in a first moment that meromorphic tensors on an algebraic curve could be easily expanded in terms of the solutions of the related Riemann monodromy problem (see e. g. [27]). However, this approach has encountered outstanding obstacles in its concrete realization. Up to now, the best way to expand meromorphic tensors and to handle their singularity structure is provided by the generalized Laurent series of [22]-[23]. To explain their usefulness with an example, one should recall that the main building block in the construction of the correlation functions of $b-c$ systems and scalar fields is the Weierstrass kernel [12]. The latter is a differential of the third kind which, on the curve, plays the same role of the Cauchy kernel on the complex plane. It is characterized by a simple pole in an assigned point and can be explicitly derived with an algorithm due to Weierstrass. However, besides the desired pole, there are also several spurious singularities, which should be eliminated by subtracting suitable counterterms [3]. A general procedure for this subtraction, which in principle strongly depends on the form of the curve, has been derived expanding the Weierstrass kernel in generalized Laurent series. As well, an operator formalism on plane algebraic curves has been established in [22]-[23] . 
If the plane curve is regarded as a Riemann surface, the generalized Laurent series can be considered as a multipoint generalization of the Krichever-Novikov bases [28] much in the spirit of [29]. On the other side, the modes entering in the expansion of meromorphic tensors consist in a finite set of tensors which are multivalued in $\mathbf{C}\left(\right.$ or $\left.\mathbf{P}^{1}\right)$. The latter have a deeper significance than modes defined on abstract Riemann surfaces, since they may be interpreted as correlators of exotic conformal field theories on the complex plane (or sphere). Theories of this kind should be both invariant under the Virasoro group of conformal transformations and under the monodromy group $\mathcal{M}$ of the original curve. The amplitudes of the class of exotic conformal field theories corresponding to $\mathcal{M}=$ $Z_{n}$ have been constructed in [30] in terms of free bosonized $b-c$ systems. Due to the above mentioned difficulties in computing partition functions, instead, only the $N$-point functions with $N>2$ can be derived if $\mathcal{M}$ is non abelian. The case $\mathcal{M}=D_{n}$ has been explicitly worked out in [31]. In this way, a concrete realization of the more general program on holonomic quantum field theories developed by Sato, Jimbo and Miwa in [32] has been achieved. The connections between generalized Laurent series and solutions of the Riemann monodromy problem have been partly explored in [31]. Some glimpses of non-commutativity in string theory have been anticipated in [33].

Despite of the above progress in understanding conformal field theories, plane algebraic curves suffer of some limitations. To obtain for instance general non-hyperelliptic Riemann surfaces of genus $g>3$, they are not powerful enough and it is necessary to consider algebraic curves in the complex projective space $\mathbf{P}^{g-1}$. Moreover, the smallest dimensional space in which a curve may be smoothly embedded is $\mathbf{P}^{3}$. In attempting to treat $b-c$ systems and scalar fields on non-plane curves, an immediate difficulty arises, because 
the analog of the Weierstrass kernel is not known. As explained before, this kernel is a crucial ingredient in the derivation of the $N$-point correlations functions and of any other meromorphic tensor. This problem is solved here in the particular case of algebraic curves in $\mathbf{P}^{3}$. A simple expression of the generalized Weierstrass kernel on these curves is obtained and, as an application, the correlation functions of the $b-c$ systems and of the scalar fields are computed on a canonical curve of genus four. Let us notice that curves in $\mathbf{P}^{3}$ include the interesting subset containing the branched covers of Riemann surfaces.

Since it is too difficult to work on a projective variety, the generalization of the Weierstrass kernel has been derived in local form after restricting oneself to local open sets of $\mathbf{P}^{3}$, where it is possible to use euclidean coordinates. This is equivalent to consider affine algebraic curves in $\mathbf{C}^{3}$. Of course, affine algebraic curves are non-compact, but it is still possible to relate them to compact Riemann surfaces by taking into account also the point at infinity and hence considering the extended complex plane $\mathbf{C} \cup \infty \equiv \mathbf{P}^{1}$. A similar strategy has been followed in the construction of the standard Weierstrass kernel, defined in [12] on affine algebraic curves in $\mathbf{C}^{2}$ or on a branched cover of $\mathbf{P}^{1}$.

The material presented in this paper is divided as follows. Section II consists in a brief and elementary introduction to the concept of algebraic curves. In Section III the derivation of the standard Weierstrass kernel on plane curves is reviewed and its main properties are discussed. A formula of Weierstrass to build a differential of the second kind on hyperelliptic curves is also presented. It is useful to recall this formula because it seems to have no track in the modern literature, but it is very important whenever current-current interactions between scalar fields have to be taken into account (see e.g. [9] and the third reference of [26]). Section IV contains the derivation of the generalized 
Weierstrass kernel and a discussion of its main properties. In general, we have been able to show that spurious poles can only appear at points in which the coordinates describing the algebraic curve in $\mathbf{C}^{3}$ become infinitely large. In Section $\mathrm{V}$ we treat the particular case of a canonical curve of genus four in details. As an application, the correlation functions of the $b-c$ systems and of the scalar fields are computed at genus four in Section VI. These theories have been already studied using several different approaches, so they provide a good testing ground for the generalized Weierstrass kernel of Section IV. Surface integrals over algebraic curves are expressed as integrals in $\mathbf{C}^{3}$ in the presence of Dirac $\delta$-functions in Appendix A. The final comments and conclusions are presented in Section VII.

\section{RIEMANN SURFACES AS ALGEBRAIC VARIETIES}

Let $\mathbf{P}^{n}$ denote the complex projective space parametrized by coordinates $\xi=$ $\xi_{0}, \ldots, \xi_{n}$. A homogeneous polynomial $F(\xi)$ of degree $d$ defines an algebraic hypersurface on $\mathbf{P}^{n}$ as the locus of the points $\xi \in \mathbf{P}^{n}$ satisfying the relation:

$$
F(\xi)=0
$$

The hypersurfaces are called quadrics if $d=2$, cubics if $d=3$, quartics if $d=4$ etc. A projective algebraic variety $\mathcal{V}$ is a subset of $\mathbf{P}^{n}$ characterized as the intersection of many hypersurfaces:

$$
\mathcal{V}=\left\{\xi \in \mathbf{P}^{n} \mid F_{1}(\xi)=\ldots=F_{k}(\xi)=0\right\}
$$

where $F_{1}, \ldots, F_{k}, k \leq n$, represent a set of homogeneous polynomials of degree $d_{1}, \ldots, d_{k}$ respectively. If $k=n-1, \mathcal{V}$ describes a projective algebraic curve $C$ in $\mathbf{P}^{n}$. A point $p \in \mathcal{V}$ 
is smooth [34] if the Jacobian matrix

$$
\mathcal{J}=\left[\frac{\partial\left(F_{1}, \ldots, F_{k}\right)}{\partial\left(\xi_{0}, \ldots, \xi_{n}\right)}\right]
$$

has

$$
\operatorname{rank}[\mathcal{J}]=k
$$

Closely related to projective algebraic varieties are the affine algebraic varieties:

$$
\mathcal{V}_{0}=\left\{x \in \mathbf{C}^{n} \mid f_{1}(x)=\ldots=f_{k}(x)=0\right\}
$$

$f_{1}, \ldots, f_{k}$ are polynomials with complex coefficients and $x=x_{1}, \ldots, x_{n}$ denotes a set of variables in the $n$-dimensional complex space $\mathbf{C}^{n}$. The definition of smooth points on affine varieties is analogous to that of smooth points on projective varieties.

It is often convenient to study projective varieties on local open patches of $\mathbf{P}^{n}$, where it is possible to use Euclidean coordinates. For instance, for $\sigma=0, \ldots, n$, one may identify $\mathbf{C}^{n}$ with the following open subsets of $\mathbf{P}^{n}[34]$ :

$$
U_{\sigma}=\left\{\left[\xi_{0}, \ldots, \xi_{n}\right] \in \mathbf{P}^{n} \mid \xi_{\sigma} \neq 0\right\}
$$

via the homeomorphism $\phi: U_{\sigma} \longrightarrow \mathbf{C}^{n}$ :

$$
\phi\left[\xi_{0}, \ldots, \xi_{n}\right]=\left(\frac{\xi_{0}}{\xi_{\sigma}}, \ldots, \frac{\xi_{\sigma-1}}{\xi_{\sigma}}, 1, \frac{\xi_{\sigma+1}}{\xi_{\sigma}}, \ldots, \frac{\xi_{n}}{\xi_{\sigma}}\right)
$$

with inverse

$$
\left(x_{1}, \ldots, x_{n}\right) \longrightarrow\left[x_{1}, \ldots, x_{\sigma}, 1, x_{\sigma+1}, \ldots, x_{n}\right]
$$


To simplify the notations, we will consider hereafter only the case $\sigma=0$. In doing that, there is no loss of generality, since none of the variables $\xi_{1}, \ldots, \xi_{n}$ plays a privileged role in the present context. Clearly, upon the identifications

$$
x_{1}=\frac{\xi_{1}}{\xi_{0}}, \ldots, x_{n}=\frac{\xi_{n}}{\xi_{0}}
$$

and

$$
f_{a}\left(x_{1}, \ldots, x_{n}\right) \equiv F_{a}\left(1, x_{1}, \ldots, x_{n}\right) \quad a=1, \ldots, k
$$

the restriction of a projective variety $\mathcal{V}$ on $U_{0}$ is equivalent to an affine variety on $\mathbf{C}^{n}$ associated to the system of equations:

$$
f_{a}\left(x_{1}, \ldots, x_{n}\right)=0 \quad a=1, \ldots, k
$$

In the special case $k=n-1$, a projective curve and the affine curve related to it via the homeomorphism (2.7) differ only by a finite number of points "at infinity". The latter are determined by the conditions $F_{1}=\ldots=F_{n-1}=\xi_{0}=0$, in which $\phi$ is no longer defined. To show that, we consider a projective algebraic curve

$$
C=\left\{\xi \in \mathbf{P}^{n} \mid F_{1}(\xi)=\ldots=F_{n-1}(\xi)=0\right\}
$$

Since the $F_{a}$ are homogeneous polynomials of degree $d_{a}, a=1, \ldots, n-1$, they can be written as follows:

$$
F_{a}(\xi)=\sum_{i_{1}, \ldots, i_{n}=0}^{d_{a}}\left(\xi_{1}\right)^{i_{1}} \ldots\left(\xi_{n}\right)^{i_{n}}\left(\xi_{0}\right)^{d_{a}-\left(i_{n}+\ldots+i_{1}\right)}
$$


If $\xi_{0}=0$, the system of algebraic equations defining $C$ is trivial unless the condition $d_{a}-\left(i_{n}+\ldots+i_{1}\right)=0$ is fulfilled. Thus, the points on the curve corresponding to $\xi_{0}=0$ are given by the residual system of equations:

$$
\sum_{i_{1}, \ldots, i_{n-1}=0}^{d_{a}}\left(\xi_{1}\right)^{i_{1}} \ldots\left(\xi_{n-1}\right)^{i_{n-1}}\left(\xi_{n}\right)^{d_{a}-\left(i_{n-1}+\ldots+i_{1}\right)}=0
$$

for $a=1, \ldots, n-1$. The above relations describe the intersection of $n-1$ hypersurfaces in $\mathbf{P}^{n-1}$, which, by Bézout theorem, contains a finite number of $d_{1} d_{2} \cdots d_{n-1}$ common points as desired.

Algebraic curves are particularly important in the study of compact Riemann surfaces. The simplest representation of a Riemann surface $\Sigma_{g}$ of genus $g$ is in terms of plane projective algebraic curves, or hypersurfaces in $\mathbf{P}^{2}$ associated to a single algebraic equation of the kind:

$$
F\left(\xi_{0}, \xi_{1}, \xi_{2}\right)=0
$$

A plane curve is said non-singular or regular provided the condition below is never verified:

$$
F=\frac{\partial F}{\partial \xi_{0}}=\frac{\partial F}{\partial \xi_{1}}=\frac{\partial F}{\partial \xi_{2}}=0
$$

Modulo conformal transformations, any compact Riemann surface coincides with a plane projective algebraic curve.

A plane curve $C$ can be projected from a point $p \notin C$ onto a complex line $L$ in $\mathbf{P}^{2}$, which does not contain $p$. After a linear change of coordinates one may take $p=[0,0,1]$ and $L=\left\{\xi \in \mathbf{P}^{2} \mid \xi_{2}=0\right\}$. The result of the projection is a representation of the Riemann surface as a branched cover (or $n$-sheeted covering) of $\mathbf{P}^{1}$, whose points are given by the 
zeros of the complex polynomial $f\left(x_{1}, x_{2}\right)=F\left(1, x_{1}, x_{2}\right)$ in the Euclidean coordinates $x_{1}=\xi_{1} / \xi_{0}$ and $x_{2}=\xi_{2} / \xi_{0}$. Solving the equation

$$
f\left(x_{1}, x_{2}\right)=0
$$

with respect to $x_{2}$, one obtains a multivalued function $x_{2}\left(x_{1}\right)$ of $x_{1} \in \mathbf{P}^{1}$. The finite branch points of $x_{2}\left(x_{1}\right)$ satisfy the relations:

$$
f\left(x_{1}, x_{2}\right)=f^{(0,1)}\left(x_{1}, x_{2}\right)=0
$$

where we have used the notation

$$
f^{(m, n)}\left(x_{1}, x_{2}\right) \equiv \frac{\partial^{m}}{\partial x_{1}^{m}} \frac{\partial^{n}}{\partial x_{2}^{n}} f\left(x_{1}, x_{2}\right)
$$

Starting from a multivalued function, a Riemann surface $\Sigma_{g}$ can be constructed in terms of sheets and cross-cuts. In analogy with eq. (2.16), a cover of $\mathbf{P}^{1}$ is non-singular if the following identities are never satisfied simultaneously:

$$
f=\frac{\partial f}{\partial x_{1}}=\frac{\partial f}{\partial x_{2}}=0
$$

The double-sheeted coverings of $\mathbf{P}^{1}$, or hyperelliptic curves, have very special properties with respect to the other curves. Their polynomial equation can always be reduced to the following one:

$$
x_{2}^{2}=P_{2 g+2}\left(x_{1}\right)
$$

where $P_{2 g+2}\left(x_{1}\right)$ is a polynomial of degree $2 g+2$ in $x_{1}$ with complex coefficients. Exploiting the group $S L(2, \mathbf{C})$ of automorphisms of the sphere $\mathbf{P}^{1}$, the number of independent coefficients reduces to $2 g-1$. Since a general Riemann surface of genus $g>2$ depends on 
$3 g-3$ complex parameters, called the moduli, not all Riemann surfaces can be hyperelliptic. As a matter of fact, hyperelliptic curves form only a subset of dimension $2 g-1$ in the moduli space [1]. On the other side, plane algebraic curves of genus $g \geq 3$ may be also non-hyperelliptic, but the number of independent parameters which is possible to accommodate in the defining polynomial $F(\xi)$ of eq. (2.15) is less than $3 g-3$ when $g>3$. For this reason, in order to construct general non-hyperelliptic Riemann surfaces, one usually considers canonical curves embedded in $\mathbf{P}^{g-1}$.

A canonical map $\varphi$ from a Riemann surface $\Sigma_{g}$ to the projective space $\mathbf{P}^{g-1}$ can be established with the help of a basis of holomorphic differentials $\omega_{1}, \ldots, \omega_{g}$ on $\Sigma_{g}$ as follows:

$$
\varphi: p \in \Sigma_{g} \longrightarrow\left[\omega_{1}, \ldots, \omega_{g}\right] \in \mathbf{P}^{g-1}
$$

If $\Sigma_{g}$ is a general non-hyperelliptic Riemann surface, $C=\varphi\left(\Sigma_{g}\right)$ is called a canonical curve. For instance, a canonical curve of genus $g=3$ is a plane projective algebraic curve of degree four. The canonical curve with $g=4$ is instead given by the complete intersection of a quadric and a cubic in $\mathbf{P}^{3}$. For $g=5$, the canonical curve is a complete intersection of three quadrics in $\mathbf{P}^{4}$ etc. A more detailed classifications of canonical curves together with a thorough discussion of some exceptional cases can be found in [4].

Here we will limit ourselves to algebraic curves in $\mathbf{P}^{3}$, which is the smallest dimensional projective space in which a curve can be smoothly embedded. In fact, smooth embeddings are not possible in $\mathbf{P}^{2}$, so that plane algebraic curves are affected by singularities at isolated points $^{2}$. Let $F(\xi)$ and $G(\xi), \xi=\xi_{0}, \xi_{1}, \xi_{2}, \xi_{3}$ be two homogeneous polynomials of

${ }^{2}$ However, a curve $C$ in $\mathbf{P}^{3}$ may always be projected in $\mathbf{P}^{2}$ in such a way that the resulting plane algebraic curve has only ordinary double points. 
degrees $d_{F}$ and $d_{G}$ respectively, whose zeros define two hypersurfaces in $\mathbf{P}^{3}$. We are mainly interested in situations in which the intersection of the two hypersurfaces is complete, i.e. they meet in a single curve $C$, whose points are given by the following system of algebraic equations:

$$
F\left(\xi_{0}, \xi_{1}, \xi_{2}, \xi_{3}\right)=G\left(\xi_{0}, \xi_{1}, \xi_{2}, \xi_{3}\right)=0
$$

We also assume that all the points of $C$ are smooth. The genus $g$ of $C$ is then given by:

$$
2 g-2=d_{F} d_{G}\left(d_{F}+d_{G}-4\right)
$$

Supposing that $\xi_{0} \neq 0$ and using the mapping (2.7), we obtain the affine algebraic variety in $\mathbf{C}^{3}$ associated to the polynomial equations:

$$
f\left(x_{1}, x_{2}, x_{3}\right)=g\left(x_{1}, x_{2}, x_{3}\right)=0
$$

Apart from the exceptional cases described in [4], the above relations describe the complete intersections of two hypersurfaces in $\mathbf{C}^{3}$. One may also view $f(x)$ and $g(x)$ as polynomials of degrees $m \leq d_{F}$ and $n \leq d_{G}$ respectively in the variable $x_{3}$. Eliminating the latter, the resultant $R\left(x_{1}, x_{2}\right)$ is a polynomial in $x_{1}$ and $x_{2}$ of degree $m n$ and the equation

$$
R\left(x_{1}, x_{2}\right)=0
$$

represents the projection of the curve (2.25) on $\mathbf{C}^{2}$. Let us notice that, with respect to the case of plane curve, this projection is not unique. For instance, it is possible to eliminate from (2.25) the variable $x_{2}$ instead of $x_{3}$. In the latter case one obtains a different resultant $R^{\prime}\left(x_{1}, x_{3}\right)$ and a different projection onto $\mathbf{C}^{2}$ :

$$
R^{\prime}\left(x_{1}, x_{3}\right)=0
$$


To (2.25) one can also associate a compact Riemann surface $\Sigma_{g}$ of genus $g$ constructed as a ramified covering of $\mathbf{P}^{1}$. For example, in the neighborhood of a point where the Jacobian

$$
J^{1}(x)=\frac{\partial f(x)}{\partial x_{2}} \frac{\partial g(x)}{\partial x_{3}}-\frac{\partial g(x)}{\partial x_{2}} \frac{\partial f(x)}{\partial x_{3}}
$$

is different from zero, $x_{1} \in \mathbf{P}^{1}$ becomes a good local coordinate and it is possible to solve the system of algebraic equations (2.25) with respect to $x_{2}$ and $x_{3}$. In this way, one obtains two multivalued functions $x_{2}\left(x_{1}\right)$ and $x_{3}\left(x_{1}\right)$, whose analytic continuation on the complex line $\mathbf{P}^{1}$ defines a Riemann surface. The ramification points are those in which the condition $J^{1}(x)=0$ is satisfied. We stress again the difference with respect to plane curves, because now there are two possible representations of $\Sigma_{g}$ in terms of branched covers of $\mathbf{P}^{1}$, corresponding to eqs. (2.26) and (2.27).

Finally, we show that eqs. (2.25) includes the branched covers of Riemann surface as a particular sub-case. Indeed, let us consider a compact Riemann surface represented as a branched cover of $\mathbf{P}^{1}$ associated to the vanishing of a polynomial:

$$
f\left(x_{1}, x_{2}\right)=0
$$

Any Riemann surface of genus $g$ can be mapped into a branched cover of this kind in a limited region of its moduli space. Solving eq. (2.29) with respect to $x_{2}$, the Riemann surface $\Sigma_{g}$ is parametrized as a curve $\mathbf{C}^{2}$ with coordinates $\left(x_{1}, x_{2}\left(x_{1}\right)\right)$. Starting from such coordinates it is still possible to realize a branched cover $\tilde{\Sigma}$ of $\Sigma_{g}$ by requiring that:

$$
g\left(x_{2}, x_{3}\right)=0
$$




\section{THE WEIERSTRASS KERNEL}

The Cauchy kernel

$$
K_{C}\left(x_{1}, x_{2}\right)=\frac{d x_{1}}{x_{1}-x_{2}}
$$

plays a fundamental role in the construction of the amplitudes of conformal field theories on the complex plane $\mathbf{C}$. This kernel has the following two properties [3]:

1) As a function of $x_{1}$, the kernel is a meromorphic differential with two simple poles in $x_{1}=x_{2}$ and in $x_{1}=\infty$. The residues are +1 and -1 respectively.

2.) As a function of $x_{2}$, the kernel is a meromorphic function with a simple pole in $x_{2}=x_{1}$ and a single zero in $x_{2}=\infty$.

A meromorphic function with a single pole cannot exist on a compact two dimensional manifold. For this reason, usually it is only required that an analogue $K(p, q) d p$ of the Cauchy kernel on a Riemann surface $\Sigma_{g}$ should have the following asymptotic behavior in a neighborhood of the point $q[3]$ :

$$
K(p, q) \sim \frac{d p}{p-q}+A(p, q)
$$

where $A(p, q)$ is finite at $p=q$.

A kernel with the above property can be constructed on $n$-sheeted coverings of $\mathbf{P}^{1}$ using a well-known algorithm of Weierstrass [12]. To this purpose, let us consider a Riemann surface $\Sigma_{g}$ represented as the locus of points defined by eq. (2.17). Solving this equation for $x_{2}$, one obtains a multivalued function $x_{2}\left(x_{1}\right)$, while $x_{1}$ is a $d$-degree mapping $x_{1}: \Sigma_{g} \longrightarrow \mathbf{P}_{1}$ from the Riemann surface to the projective sphere $\mathbf{P}^{1}$. Any point $p \in \Sigma_{g}$ is in a $1-1$ correspondence with a point on the branched cover $x(p) \equiv x_{1}(p), x_{2}\left(x_{1}(p)\right)$. 
Let us put now $y(q) \equiv x_{1}(q), x_{2}\left(x_{1}(q)\right)$, where $q \neq p$. Then an analogue of the Cauchy kernel on the branched cover is the following Weierstrass kernel:

$$
K_{W}(x, y)=\frac{f\left(y_{1}, x_{2}\right)}{\left(x_{2}-y_{2}\right) f^{(0,1)}\left(x_{1}, x_{2}\right)} \frac{d x_{1}}{x_{1}-y_{1}}
$$

To study its behavior in the limit $x_{1} \longrightarrow y_{1}$, one can expand $f\left(y_{1}, x_{2}\right)$ in series of Taylor near the point $x_{2}=y_{2}$ :

$$
f\left(y_{1}, x_{2}\right) \sim f\left(y_{1}, y_{2}\right)+f^{(0,1)}\left(y_{1}, y_{2}\right)\left(x_{2}-y_{2}\right)+\ldots
$$

Since $f\left(y_{1}, y_{2}\right)=0$, it is clear that the Weierstrass kernel satisfies requirement (3.2):

$$
K_{W}(x, y) \sim \frac{d x_{1}}{x_{1}-y_{1}}
$$

The next term in the Taylor expansion gives in fact a contribution proportional to

$$
A\left(x_{1}, y_{1}\right)=\frac{f^{(0,2)}\left(y_{1}, y_{2}\right) f^{(1,0)}\left(x_{1}, x_{2}\right)}{f^{(0,1)}\left(x_{1}, x_{2}\right)} d x_{1}
$$

which is finite when $x_{1}=y_{1}$.

One should also consider those points $p \neq q \in \Sigma_{g}$ which, on the branched cover, correspond to coordinates $x(p)$ and $y(q)$ such that $x_{1}(p)=x_{1}(q)$ and $x_{2}\left(x_{1}(p)\right) \neq x_{2}\left(x_{1}(q)\right)$. In this case, there is no spurious pole in the Weierstrass kernel despite the presence of the factor $\left(x_{1}-y_{1}\right)^{-1}$. As a matter of fact, expanding the function $f\left(y_{1}, x_{2}\right)$ in series of Taylor in $y_{1}$ :

$$
f\left(y_{1}, x_{2}\right)=f\left(x_{1}, x_{2}\right)+f^{(1,0)}\left(x_{1}, x_{2}\right)\left(x_{1}-y_{1}\right)+\ldots
$$

and substituting in (3.3) one obtains

$$
K_{W}(x, y) \sim \frac{f^{(1,0)}\left(x_{1}, x_{2}\right)}{\left(x_{2}-y_{2}\right) f^{(0,1)}\left(x_{1}, x_{2}\right)} d x_{1}
$$


which is finite since $x_{2}-y_{2} \neq 0$ by hypothesis.

One may also check that the Weierstrass kernel has no spurious poles at the branch points if the curve is regular. To this purpose it is possible to exploit the relation

$$
\frac{d x_{1}}{f^{(0,1)}\left(x_{1}, x_{2}\right)}=\frac{d x_{2}}{f^{(1,0)}\left(x_{1}, x_{2}\right)}
$$

which is a consequence of the implicit function theorem [34]. Let us now consider a branch point $x_{1}=x_{1}(a)$ of multiplicity $\nu$, where $\nu$ is an integer and $a \in \Sigma_{g}$. We suppose for the moment that $x_{2}\left(x_{1}(a)\right)$ is finite. Near the branch point we choose a good local coordinate $t$ such that:

$$
x_{1}-x_{1}(a)=t^{\nu}
$$

Since $x_{2}\left(x_{1}(a)\right)$ is not divergent, its approximate expansion in powers of $t$ will look as follows:

$$
x_{2} \sim \alpha_{0}+\alpha_{1} t+\alpha_{2} t^{2}+\ldots
$$

with $\alpha_{0,1,2}$ being constants. Thus $d x_{1} \sim \nu t^{\nu-1} d t$ and $d x_{2} \sim d t$. Remembering that the function $f^{(1,0)}\left(x_{1}, x_{2}\right)$ does not vanish at a branch point due to the regularity hypothesis (2.4), we find that near $x_{1}(a)$ eq. (3.9) is approximated by:

$$
\frac{d x_{1}}{f^{(0,1)}\left(x_{1}, x_{2}\right)} \sim d t
$$

This shows that the zeros of $f^{(0,1)}\left(x_{1}, x_{2}\right)$ are absorbed by the corresponding zeros of the differential $d x_{1}$, so that the Weierstrass kernel cannot be singular at the finite branch points. If $x_{2}$ has a pole of order $k$ near a branch point, instead, it is always possible to perform the change of variables

$$
x_{2}^{\prime}=\left(x_{1}-x_{1}(a)\right)^{k} x_{2}
$$


In the new coordinates, $x_{2}^{\prime}$ remains finite at the branch point $x_{1}(a)$ and the above demonstration applies again.

Of course, on a Riemann surface a single pole is not allowed, so that the Weierstrass kernel (3.3) must contain also other spurious poles both as a function of $x_{1}$ and $y_{1}$. Typically, they appear whenever the variables $x_{1}, x_{2}$ and $y_{1}, y_{2}$ become infinitely large. A general procedure to subtract these spurious poles while keeping the property (3.2) has been already discussed in ref. [22]-[23] The amplitudes of some free conformal field theories on branched covers of $\mathbf{P}^{1}$ have been constructed in.

To conclude this Section, we present a beautiful formula of Weierstrass [12] to construct a second kind differential on an hyperelliptic curve. Thus, we consider curves of the kind (2.21), where

$$
P_{2 g+2}\left(x_{1}\right)=A_{0}+A_{1} x_{1}+\ldots+A_{2 g+2} x^{2 g+2}
$$

Let us define the function:

$$
\begin{aligned}
& R\left(x_{1}, x_{1}^{\prime}\right)=A_{0}+\frac{1}{2} A_{1}\left(x_{1}+x_{1}^{\prime}\right)+A_{2} x_{1} x_{1}^{\prime}+\frac{1}{2} A_{3}\left(x_{1}+x_{1}^{\prime}\right) x_{1} x_{1}^{\prime}+A_{4}\left(x_{1} x_{1}^{\prime}\right)^{2}+ \\
& +\frac{1}{2} A_{5}\left(x_{1}+x_{1}^{\prime}\right)\left(x_{1} x_{1}^{\prime}\right)^{2}+\ldots+\frac{1}{2} A_{2 g+1}\left(x_{1}+x_{1}^{\prime}\right)\left(x_{1} x_{1}^{\prime}\right)^{g}+A_{2 g+2}\left(x_{1} x_{1}^{\prime}\right)^{g+1}
\end{aligned}
$$

Clearly, $R\left(x_{1}, x_{1}^{\prime}\right)$ satisfies the property

$$
R\left(x_{1}, x_{1}^{\prime}\right)=R\left(x_{1}^{\prime}, x_{1}\right)
$$

Moreover, if $x_{1}=x_{1}^{\prime}$, one has that:

$$
R\left(x_{1}, x_{1}^{\prime}\right)=\left.P_{2 g+2}\left(x_{1}\right) \quad \frac{\partial R\left(x_{1}, x_{1}^{\prime}\right)}{\partial x_{1}}\right|_{x_{1}=x_{1}^{\prime}}=\frac{1}{2} \frac{\partial P_{2 g+2}\left(x_{1}\right)}{\partial x_{1}}
$$


The differential of the second kind is given by:

$$
\tau_{x_{1}^{\prime}}\left(x_{1}\right)=-\frac{y\left(x_{1}\right) y^{\prime}\left(x_{1}\right)+R\left(x_{1}, x_{1}^{\prime}\right)}{2\left(x_{1}-x_{1}^{\prime}\right)^{2} y\left(x_{1}\right) y^{\prime}\left(x_{1}\right)} d x_{1}
$$

where $y^{\prime}\left(x_{1}\right)=\frac{\partial y\left(x_{1}\right)}{\partial x_{1}}$. It is easy to show using the properties $(3.16)-(3.17)$ that $\tau_{x_{1}^{\prime}}\left(x_{1}\right)$ has only a pole of the second order in $x_{1}=x_{1}^{\prime}$ as it should be. Unfortunately it is not simple to extend the elegant formula (3.18) to the $Z_{n}$ symmetric curves, not to mention the general plane algebraic curves of eq. (2.17) or the even more complicated curves (2.25).

\section{GENERALIZED WEIERSTRASS KERNELS}

In this Section we construct analogues of the Weierstrass kernel on affine algebraic curves defined by the system of equations (2.25). Even if it will not be strictly necessary, we suppose to fix the ideas that the intersection of the two hypersurfaces in (2.25) is complete and gives as a result an algebraic curve $C$ which coincides, modulo conformal transformations, with a Riemann surface $\Sigma$.

The following two different cases can formally be treated in the same way. On one side, $x_{1}, x_{2}, x_{3}$ may be interpreted as coordinates in $\mathbf{C}^{3}$, so that $\Sigma$ is not compact due to the absence of the points at infinity. Alternatively, the vanishing of the polynomials (2.25) can be associated to a ramified covering of $\mathbf{P}^{1}$ as we have seen in Section II and $\Sigma$ is a compact Riemann surface of genus $g$.

Let us consider as in the previous Section two different points $p, q \in \Sigma$. On the algebraic curve $C$ they correspond to coordinates $x(p)=x_{1}(p), x_{2}(p), x_{3}(p)$ and $y(q)=$ $y_{1}(q), y_{2}(q), y_{3}(q)$. A possible analogue of the Weierstrass kernel on $C$ is given by:

$$
K_{\mathrm{sym}}(x, y)=\frac{1}{3} \sum_{i=1}^{3} \frac{N^{i}(x, y)}{J^{i}(x)} \frac{d x_{i}}{\left(x_{1}-y_{1}\right)\left(x_{2}-y_{2}\right)\left(x_{3}-y_{3}\right)}
$$


where

$$
J^{i}(x)=\epsilon^{i k l} \frac{\partial f(x)}{\partial x_{k}} \frac{\partial g(x)}{\partial x_{l}}
$$

and

$$
\begin{aligned}
& N^{1}(x, y)=f\left(y_{1}, y_{2}, x_{3}\right) g\left(x_{1}, y_{2}, x_{3}\right)-f\left(x_{1}, y_{2}, x_{3}\right) g\left(y_{1}, y_{2}, x_{3}\right) \\
& N^{2}(x, y)=f\left(x_{1}, y_{2}, y_{3}\right) g\left(x_{1}, x_{2}, y_{3}\right)-f\left(x_{1}, x_{2}, y_{3}\right) g\left(x_{1}, y_{2}, y_{3}\right) \\
& N^{3}(x, y)=f\left(y_{1}, x_{2}, y_{3}\right) g\left(y_{1}, x_{2}, x_{3}\right)-f\left(y_{1}, x_{2}, x_{3}\right) g\left(y_{1}, x_{2}, y_{3}\right)
\end{aligned}
$$

Here $\epsilon^{i k l}$ denotes the completely antisymmetric tensor in three dimensions with the convention $\epsilon^{123}=1$. We note that the variables $x, y$ and the functions $f$ and $g$ enter symmetrically in the expression of the kernel (4.1), as it should be since none of them plays a privileged role in the definition of the algebraic curve. The symmetry under the exchange of $f$ and $g$ is also related to the freedom of projecting the curve in the two possible ways shown by eqs. (2.26) and (2.27).

Equivalent kernels can be obtained starting from $K_{\mathrm{sym}}(x, y)$ and adding differentials in such a way that the behavior near the singularity in $x=y$ remains unchanged. For instance, exploiting the identities:

$$
\frac{d x_{1}}{J^{1}}=\frac{d x_{2}}{J^{2}}=\frac{d x_{3}}{J^{3}}
$$

which are a consequence of the implicit function theorem and using the fact that the numerators $N^{1}, N^{2}, N^{3}$ differ each other by functions that vanish in $x=y$, one may derive the following kernel:

$$
K(x, y)=\frac{N^{1}(x, y)}{J^{1}(x)} \frac{d x_{1}}{\left(x_{1}-y_{1}\right)\left(x_{2}-y_{2}\right)\left(x_{3}-y_{3}\right)}
$$


The above kernel is less symmetric than $K_{\mathrm{sym}}(x, y)$, but has a more compact expression. In the particular case of a branched cover of a plane curve, in which the algebraic equations (2.25) assume the form (2.29) and (2.30), $K(x, y)$ is simply given by:

$$
K(x, y)=-\frac{f\left(x_{1}, y_{2}\right) g\left(y_{2}, x_{3}\right)}{G_{x_{3}}(x) F_{x_{2}}(x)} \frac{1}{\left(x_{1}-y_{1}\right)\left(x_{2}-y_{2}\right)\left(x_{3}-y_{3}\right)}
$$

Let us investigate the behavior of $K(x, y)$ near $x=y$. To this purpose, it is sufficient to expand $N^{1}(x, y)$ with respect to $y$ at the point $x$. At the leading order:

$$
N^{1}(x, y) \sim J^{1}(x) \Delta x_{2} \Delta x_{3}
$$

where $\Delta x_{i}=y_{i}-x_{i}$ is very small by hypothesis. We note that, in principle, there are also contributions proportional to $\Delta x_{1}$ in the expansion of $N^{1}(x, y)$. In order to obtain eq. (4.9), $\Delta x_{1}$ has been expressed in terms of $\Delta x_{2}$ and $\Delta x_{2}$ with the help of (4.6). Substituting (4.9) in (4.7) we find that $K(x, y)$ satisfies the property (3.2) as desired:

$$
K(x, y) \sim \frac{d x_{1}}{\left(x_{1}-y_{1}\right)}
$$

A similar calculation for $K_{\mathrm{sym}}(x, y)$ gives the result

$$
K_{\mathrm{sym}}(x, y) \sim \frac{1}{3} \sum_{i=1}^{3} \frac{d x_{i}}{\left(x_{i}-y_{i}\right)}
$$

In this case one should remember that not all variables $x_{1}, x_{2}, x_{3}$ are independent due to the relations (2.25), so that eq. (4.11) must be worked out further. Assuming for instance that $x_{1}$ is a good local coordinate in a neighborhood of the point $p$, one can solve the system of algebraic equations (2.25) with respect to the remaining variables, so that $x_{j}=x_{j}\left(x_{1}\right)$ 
for $j=2,3$. In the same way $y_{j}=x_{j}\left(y_{1}\right)$ for $j=2,3$ in a neighborhood of $q$, with $y_{1}=x_{1}+\Delta x_{1}$. As a consequence

$$
d x_{j}\left(x_{1}\right)=\frac{d x_{j}}{d x_{1}} d x_{1} \quad x_{j}\left(y_{1}\right)-x_{j}\left(x_{1}\right) \sim-\frac{d x_{j}}{d x_{1}} \Delta x_{1} \quad j=2,3
$$

Using the above relations in (4.11), one obtains:

$$
K_{\mathrm{sym}}(x, y) \sim \frac{d x_{1}}{\left(x_{1}-y_{1}\right)}
$$

Thus, also the kernel (4.1) has the requested behavior near the pole in $x=y$.

Besides the required simple pole in $x=y$, the kernels (4.1) and (4.7) have also spurious poles, which have to be controlled and suitably subtracted in order to construct physical correlation functions with desired singularities. The study of these spurious poles will be the subject of the rest of this Section.

Since the structure of the kernels (4.1) and (4.7) consists in ratios of polynomials of $x$ and $y$, their possible divergences may only occur at the zeros of the denominators $J^{i}(x)\left(x_{1}-y_{1}\right)\left(x_{2}-y_{2}\right)\left(x_{3}-y_{3}\right)$ or at the infinities of the numerators $N^{i}(x, y), i=1,2,3$.

First of all, we consider the zeros of $J^{1}(x)$. The cases in which $i=2,3$ can be treated in an analogous way. Let $x(a)=\left(x_{1}(a), x_{2}(a), x_{3}(a)\right), a \in \Sigma$, be a point on $C$ for which $J^{1}(x)=0$. In $x(a)$ the system of algebraic equations (2.25) becomes no longer invertible with respect to $x_{2}$ and $x_{3}$. Given a good local coordinate $t$ in a neighborhood of $x(a)$, this implies that

$$
x_{1}-x_{1}(a)=t^{\lambda}
$$

and

$$
x_{2}=\alpha_{0}+\alpha_{1} t^{\mu}+\ldots \quad x_{3}=\beta_{0}+\beta_{1} t^{\nu}+\ldots
$$


where $\lambda$ is an integer containing the integers $\mu$ and $\nu$ as sub-factors. In eq. (4.15) we suppose that $x_{2}$ and $x_{3}$ do not diverge in $a$, so that $\lambda, \mu, \nu$ are all positive. If not, it is always possible to perform in eq. (2.25) a change of variables $x_{2}, x_{3} \rightarrow x_{2}^{\prime}, x_{3}^{\prime}$ similar to that of eq. (3.13) to make the new variables $x_{2}^{\prime}, x_{3}^{\prime}$ finite in $a$. We note that the monodromy properties of $x_{2}$ and $x_{3}$ may be in general different, so that $\mu$ and $\nu$ need not to be equal. Near $x(a)$ the relations (4.6) become:

$$
\frac{\lambda t^{\lambda-1} d t}{J^{1}(x)}=\frac{\mu t^{\mu-1} d t}{J^{2}(x)}=\frac{\nu t^{\nu-1} d t}{J^{3}(x)}
$$

where $x$ is a function of $t$ given by eqs. (4.14) and (4.15). At this point it is possible to invoke the regularity condition (2.4), which assures that $J^{2}(x), J^{3}(x) \neq 0$ in $x=x(a)$. Since $\mu, \nu \leq \lambda$ it is easy to see from (4.16) that the zeros of $J^{1}(x)$ are absorbed by the corresponding zeros of the differential $d x_{1}$. As a consequence, there are no spurious divergences at these points.

One can also verify that there are no poles when $\left(x_{1}-y_{1}\right)\left(x_{2}-y_{2}\right)\left(x_{3}-y_{3}\right)=0$ apart from the one in $x(p)=y(q)$, which is related to the required singularity in $p=q$. Spurious poles of this kind may in principle occur if two different points $p, q \in \Sigma$ correspond on the algebraic curve to coordinates $x(p)$ and $x(q)$ characterized by the fact that some of their components, but not all, coincide (for instance $x_{1}=y_{1}, x_{2}=y_{2}$ and $x_{3} \neq y_{3}$ ). The proof that no spurious divergence arises in this case is straightforward and will not be reported here.

In conclusion, the kernels (4.1) and (4.7) diverge only at the poles of the numerators $N^{i}(x, y)$. In general, the latter are located at the points in which the variables $x$ and $y$ become very large. 


\section{THE CASE OF GENERAL NON-HYPERELLIPTIC CURVES OF GENUS FOUR}

A general non-hyperelliptic algebraic curve of genus four is given by the complete intersection of a quadric with a cubic as mentioned in Section II. In fact, putting $d_{F}=3$ and $d_{G}=2$ in (2.24), one obtains exactly $g=4$. For instance. one can choose in eqs. $(2.23) G(\xi)$ as follows:

$$
G\left(\xi_{1}, \xi_{2}, \xi_{3}, \xi_{4}\right)=\xi_{0} \xi_{1}-\xi_{2} \xi_{3}
$$

while $F(\xi)$ is a homogeneous polynomial of degree three. In affine coordinates $\xi_{i}=\xi_{i} / \xi_{0}$, $i=1,2,3, F(\xi)$ and $G(\xi)$ are replaced by:

$$
G\left(1, x_{1}, x_{2}, x_{3}\right)=g\left(x_{1}, x_{2}, x_{3}\right)=x_{2} x_{3}-x_{1}
$$

$$
F\left(1, x_{1}, x_{2}, x_{3}\right)=f\left(x_{1}, x_{2}, x_{3}\right)=x_{3}^{3}+h_{1}\left(x_{1}, x_{2}\right) x_{3}^{2}+h_{2}\left(x_{1}, x_{2}\right) x_{3}+h_{3}\left(x_{1}, x_{2}\right)
$$

where

$$
h_{i}\left(x_{1}, x_{2}\right)=\sum_{\substack{k, l=0 \\ k+l \leq 3}}^{i} a_{k l}^{(i)} x_{1}^{k} x_{2}^{l} \quad i=1,2,3
$$

and the $a_{k l}^{(i)}$ are complex coefficients. We note that the polynomial $f\left(\xi_{1}, \xi_{2}, \xi_{3}\right)$ has been ordered according to the different powers of $x_{3}$. This is just a convention which does not reflect any special role of $x_{3}$. In the same way one could order $f\left(\xi_{1}, \xi_{2}, \xi_{3}\right)$ with respect to the powers of $x_{1}$ or $x_{2}$. All necessary ingredients to construct the Weierstrass kernels (4.1) and (4.7) are derived in a straightforward way substituting eqs. (5.2) and (5.3) in (4.2)-(4.5) 
In the following we will assume that $x_{1}$ is a good local coordinate of the curve. The cases in which $x_{1} \in \mathbf{C}$ or $x_{1} \in \mathbf{P}_{1}$ can be formally treated in the same way. Accordingly, we solve the system of algebraic equations

$$
f\left(x_{1}, x_{2}, x_{3}\right)=g\left(x_{1}, x_{2}, x_{3}\right)=0
$$

with respect to $x_{1}^{3}$. As a result, one obtains two multivalued functions $x_{2}\left(x_{1}\right)$ and $x_{3}\left(x_{1}\right)$. Due to eq. (5.2), both $x_{2}\left(x_{1}\right)$ and $x_{3}\left(x_{1}\right)$ share the same monodromy properties. They define a Riemann surface $\Sigma_{4}$ constructed in terms of sheets. It is easy to check that $x_{2}\left(x_{1}\right)$ and $x_{3}\left(x_{1}\right)$ have six branches, so that $\Sigma_{4}$ consists of six sheets glued together at the branch lines. The computation of the resultant $R\left(x_{1}, x_{2}\right)$ of the two algebraic equations (5.5) is straightforward and gives the following plane curve equation associated to $\Sigma_{4}$ :

$$
x_{1}^{3}+h_{1}\left(x_{1}, x_{2}\right) x_{1}^{2} x_{2}+h_{2}\left(x_{1}, x_{2}\right) x_{1} x_{2}^{2}+h_{3}\left(x_{1}, x_{2}\right) x_{2}^{3}=0
$$

which indeed describes a curve of genus four.

Due to the peculiar role played by the variable $x_{1}$, it is natural to consider the kernel $K(x, y)$ instead of the more symmetric one of eq. (4.1). Inserting eqs. (5.2) and (5.3) in (4.7) one obtains:

$$
K(x, y)=-\frac{d x_{1}}{\left(x_{1}-y_{1}\right) J^{1}(x)}\left[\frac{x_{3} f\left(y_{1}, y_{2}, x_{3}\right)}{\left(x_{3}-y_{3}\right)}+\frac{y_{2} f\left(x_{1}, y_{2}, x_{3}\right)}{\left(x_{2}-y_{2}\right)}\right]
$$

where we should remember that we are dealing with multivalued functions $x_{j}=x_{j}\left(x_{1}\right)$ and $y_{j}=y_{j}\left(y_{1}\right)$ for $j=2,3$. With respect to the general formula (4.7), in this case some

3 There is no loss of generality in doing that. If one wishes to study the algebraic curve in the neighborhood of a branch point, where $x_{1}$ is no longer a good coordinate, it is always possible to perform a conformal transformation and to consider $x_{1}$ as a function of $x_{2}$ or $x_{3}$. 
simplifications have been possible because of the particular form of $g\left(x_{1}, x_{2}, x_{3}\right)$. One can check that the above kernel has the desired pole when $x_{i}=y_{i}$ for $i=1,2,3$. If one wishes to study the kernel (5.7) in the neighborhood of a branch point where $J^{1}(x)=0$, it is possible to perform the conformal transformation $x_{1}=x_{1}\left(x_{2}\right)$.

Let us now concentrate on the spurious divergences of $K(x, y)$. From the previous Section, we know that they may only occur at the infinities of the variables $x_{i}, y_{i}, i=1,2,3$. In this case, the situation is made simpler by the fact that $x_{2}\left(x_{1}\right)$ and $x_{3}\left(x_{1}\right)$ have no poles for finite values of $x_{1}$. To show that, let us imagine that a point $a \in \Sigma_{4}$ corresponds on the algebraic curve to a point $x_{1}(a)$ where $x_{3}$ has a pole of order $s$ :

$$
x_{3}\left(x_{1}\right) \sim\left(x_{1}-x_{1}(a)\right)^{-s}
$$

Due to eq. (5.2), the function $x_{2}\left(x_{1}\right)$ has a zero of the same order at the same point:

$$
x_{2}\left(x_{1}\right) \sim\left(x_{1}-x_{1}(a)\right)^{s}
$$

Thus, in the limit $x_{1}=x_{1}(a)$ the polynomials $h_{i}\left(x_{1}, x_{2}\right), i=1,2,3$, may be replaced by suitable constants $A_{1}, A_{2}, A_{3}$ neglecting higher order terms in $x_{1}-x_{1}(a)$. Hence, eq. (5.3) is approximated by:

$$
x_{3}^{3}+A_{1} x_{3}^{2}+A_{2} x_{3}+A_{3}=0
$$

Clearly, the above equation has no solutions if $x_{3}=\infty$. Analogously, since $x_{3}$ and $x_{2}$ enter in eq. (5.5) symmetrically, it is possible to verify that $x_{2}\left(x_{1}\right)$ has no divergences for finite values of $x_{1}$. 
To study the singularities of $x_{2}\left(x_{1}\right)$ and $x_{3}\left(x_{1}\right)$ at infinity, it is convenient to introduce the new variable $x_{1}^{\prime}=x_{1}^{-1}$. Let us now suppose that $x_{2}$ and $x_{3}$ have the following behavior near $x_{1}^{\prime}=0$ :

$$
x_{2}=\alpha\left(x_{1}^{\prime}\right)^{s}+\ldots \quad x_{3}=\beta\left(x_{1}^{\prime}\right)^{-1-s}+\ldots
$$

The second relation (5.11) is again a consequence of (5.2). Substituting the ansatz (5.11) in (5.3), it is easy to verify that the latter equation is satisfied only if $s=-1$ or $s=0$. In the first case, there are three branches of $x_{2}$ and $x_{3}$ such that $x_{2}$ diverges:

$$
x_{2} \sim \frac{1}{x_{1}^{\prime}} \quad x_{3} \sim \text { const }
$$

If $s=0$, instead, there are other three branches in which $x_{3}$ becomes singular:

$$
x_{2} \sim \text { const } \quad x_{3} \sim \frac{1}{x_{1}^{\prime}}
$$

At this point, we are ready to discuss the spurious poles of the kernel (5.7). As a meromorphic differential in $x_{1}, K(x, y)$ has three simple poles in $x_{1}=0$. The latter occur in the three branches of $x_{2}$ and $x_{3}$ where eq. (5.13) is satisfied. In each of these branches, $K(x, y)$ has residue $-\frac{1}{3}$ :

$$
K(x, y)=-\frac{1}{3} \frac{d x_{1}^{\prime}}{x_{1}^{\prime}}+\ldots
$$

It is easy to check that there are no other spurious singularities in $x^{4}$. Thus $K(x, y)$ is a differential of the third kind on $\Sigma_{4}$. Taking into account also the simple pole in $x=y$, the sum of all its residues vanishes as it should be on a compact surface.

4 In principle one would expect the appearance of singularities also in the branches in which eq. (5.12) is satisfied due to the symmetry between the variables $x_{2}$ and $x_{3}$ in (5.5). However, we remember that this symmetry has been explicitly broken by he way in which the kernel $k(x, y)$ has been constructed. 
To study the singularities with respect to the variables $y$, it is convenient to rewrite $K(x, y)$ in a slightly different form, obtained by expanding in (5.7) $f\left(y_{1}, y_{2}, x_{3}\right)$ and $f\left(x_{1}, y_{2}, x_{3}\right)$ in powers of $x_{3}$ and $y_{2}$ respectively. Since $f(x)$ is a polynomial in its arguments of degree three, the expansions below:

$$
\begin{aligned}
& f\left(y_{1}, y_{2}, x_{3}\right)=\sum_{n=1}^{3} \frac{\partial^{n} f(y)}{\partial y_{3}^{n}} \frac{\left(x_{3}-y_{3}\right)^{n}}{n !} \\
& f\left(x_{1}, y_{2}, x_{3}\right)=\sum_{n=1}^{3} \frac{\partial^{n} f(x)}{\partial x_{2}^{n}} \frac{\left(x_{2}-y_{2}\right)^{n}}{n !}
\end{aligned}
$$

are exact. As a consequence, inserting (5.15) and (5.16) in (5.7), we have that

$$
K(x, y)=\frac{P(x, y)}{J^{1}(x)\left(x_{1}-y_{1}\right)} d x_{1}
$$

where

$$
P(x, y)=\sum_{n=1}^{3}\left[y_{2} \frac{\partial^{n} f(x)}{\partial x_{2}^{n}} \frac{\left(x_{2}-y_{2}\right)^{n-1}}{n !}-x_{3} \frac{\partial^{n} f(y)}{\partial y_{3}^{n}} \frac{\left(x_{3}-y_{3}\right)^{n-1}}{n !}\right]
$$

Now we exploit the fact that the spurious divergences of $K(x, y)$ are located at the points in which $y_{1}=\infty$ as the previous analysis has shown. Therefore, it is convenient to keep in the kernel only the contributions which diverge in $y_{1}=\infty$. Using the formula $\frac{1}{x_{1}-y_{1}}=-\frac{1}{y_{1}} \sum_{n=0}^{\infty}\left(\frac{x_{1}}{y_{1}}\right)^{n}$, we find:

$$
K(x, y) \sim \frac{d x_{1}}{J^{1}(x)}\left[\mathcal{A}_{1}(y)+x_{1} \mathcal{A}_{2}(y)+x_{2} \mathcal{A}_{3}(y)+x_{3} \mathcal{A}_{4}(y)\right]
$$

where

$$
\begin{gathered}
\mathcal{A}_{1}(y)=-y_{1}^{-1}\left(a_{03}^{(3)} y_{2}^{3}+a_{02}^{(3)} y_{2}^{2}\right) \\
\mathcal{A}_{2}(y)=-a_{03}^{(3)} \frac{y_{2}^{3}}{y_{1}^{2}}-a_{12}^{(3)} \frac{y_{2}^{2}}{y_{1}}
\end{gathered}
$$




$$
\begin{gathered}
\mathcal{A}_{3}(y)=-a_{03}^{(3)} \frac{y_{2}^{2}}{y_{1}} \\
\mathcal{A}_{4}(y)=y_{1}^{-1}\left[-a_{02}^{(2)} y_{2}^{2}+7 y_{3}^{2}+3 y_{3} h_{1}\left(y_{1}, y_{2}\right)+h_{2}\left(y_{1}, y_{2}\right)\right]
\end{gathered}
$$

We notice that $K(x, y)$ has the following behavior near the spurious poles in $y_{1}=\infty$ :

$$
K(x, y) \sim \sum_{i=1}^{4} \omega_{i}(x) \mathcal{A}_{i}(y)
$$

where the $\omega_{i}(x)$ are holomorphic differentials. As a matter of fact, using eqs. (5.12) and (5.13) it is easy to check that a basis of holomorphic differentials on $\Sigma_{4}$ is:

$$
\begin{array}{ll}
\omega_{1}(x)=\frac{d x_{1}}{J^{1}(x)} & \omega_{2}(x)=\frac{x_{1} d x_{1}}{J^{1}(x)} \\
\omega_{3}(x)=\frac{x_{2} d x_{1}}{J^{1}(x)} & \omega_{4}(x)=\frac{x_{3} d x_{1}}{J^{1}(x)}
\end{array}
$$

As a result, it is possible to conclude that the divergent part of $K(x, y)$ given in eq. (5.19) is proportional to holomorphic differentials in $x$. This property of the kernel (5.7) will be crucial in subtracting the spurious divergences from the amplitudes of the conformal field theories which will be the subject of the next Section.

\section{FREE CONFORMAL FIELD THEORIES ON A GENERAL NON- HYPERELLIPTIC CURVE OF GENUS FOUR}

In this Section we apply the previous results to the computation of the amplitudes of the free conformal field theories appearing in the action of bosonic strings on a general non-hyperelliptic surface of genus four $\Sigma_{4}$. These systems are well known and represent a good way to test the generalized Weierstrass differential constructed in Section IV. Apart from the above mentioned works in the case of hyperelliptic and non-hyperelliptic curves, 
they have been studied by various authors and different methods on Riemann surfaces (see for instance $[35]-[42])$.

First of all, we discuss the case of fermionic $b-c$ systems with integer $\operatorname{spin} \lambda=1,2$ In isothermal coordinates $p, \bar{p}$ where the metric becomes conformally flat, their action is given by:

$$
S_{b c}=\int_{\Sigma_{4}} d^{2} p b \bar{\partial} c
$$

where $d^{2} p=i d p \wedge d \bar{p}$ and $\bar{\partial}=\frac{\partial}{\partial \bar{p}}$. The fields $b$ are meromorphic tensors on $\Sigma_{4}$ with $\lambda$ lower indices, while the fields $c$ are characterized by $\lambda-1$ upper indices. From eq. (6.1) one obtains the following equations of motion:

$$
\bar{\partial} b=\bar{\partial} c=0
$$

We start with the case $\lambda=2$. We denote with $\phi_{\mu}, \mu=1, \ldots, 9$, the holomorphic quadratic differentials which represent a basis of non-trivial solutions of (6.2). If $p_{1} \ldots p_{m}$ and $q_{1} \ldots q_{n}$ are points in $\Sigma_{4}$, the nonvanishing correlation functions of the $b-c$ systems

$$
\mathcal{G}_{2}(p ; q)=\left\langle\prod_{\alpha=1}^{m} b\left(p_{\alpha}\right) \prod_{\beta=1}^{n} c\left(q_{\alpha}\right)\right\rangle
$$

should satisfy the relation $m-n=3 g-3=9$.

The zeros and poles of $\mathcal{G}_{2}(p ; q)$ are determined by the physical properties of the fields. To specify their locations and orders it is convenient to introduce the concept of divisor. Let $\Delta[T]$ denote the divisor of a given meromorphic tensor $T(p)$ on the Riemann surface. If $T$ has zeros at $p=p_{i}$ of order $\mu_{i}, i=1, \ldots, m_{z e r o s}$ and poles of order $\nu_{j}$ at $p=q_{j}$, $j=1, \ldots, n_{\text {poles }}$, then $\Delta[T]$ can be written as follows:

$$
\Delta[T]=\sum_{i=1}^{m_{\text {zeros }}} \mu_{i} p_{i}-\sum_{j=1}^{n_{\text {poles }}} \nu_{j} q_{j}
$$


The correlation function (6.3) is a tensor with two lower indices in each variable $p_{\alpha}, \alpha=$ $1, \ldots, m$ and the following divisor:

$$
\Delta_{p_{\alpha}}\left[\mathcal{G}_{2}\right]=\sum_{\substack{\alpha^{\prime}=1 \\ \alpha^{\prime} \neq \alpha}}^{m} p_{\alpha^{\prime}}-\sum_{\substack{\beta^{\prime}=1 \\ \beta^{\prime} \neq \beta}}^{n} q_{\beta^{\prime}}
$$

With respect to $q_{\beta}$, instead, $\mathcal{G}_{2}(p ; q)$ is a vector with an upper index and divisor:

$$
\Delta_{q_{\beta}}\left[\mathcal{G}_{2}\right]=\sum_{\substack{\beta^{\prime}=1 \\ \beta^{\prime} \neq \beta}}^{n} q_{\beta^{\prime}}-\sum_{\substack{\alpha^{\prime}=1 \\ \alpha^{\prime} \neq \alpha}}^{m} p_{\alpha^{\prime}}
$$

To construct $\mathcal{G}_{2}(p ; q)$ explicitly, we represent $\Sigma_{4}$ as a ramified covering $C_{4}$ associated to the system of algebraic equations (5.5) treated in the previous Section. We begin by noting that a meromorphic tensor $T(p)$ with $\lambda$ indices on the Riemann surface $\Sigma_{4}$ corresponds in $C_{4}$ to a tensor $T(x(p))=T_{x_{1} \ldots x_{1}}(x(p)) d x_{1}^{\lambda}$, where $x(p)=x_{1}(p), x_{2}(p), x_{3}(p)$ and $x_{1}(p) \in \mathbf{P}_{1}$. A pole or a zero of $T(p)$ in $p=q$ corresponds to a pole or a zero of $T(x(p))$ of the same order in $x(p)=y(q)$.

A basis of independent holomorphic quadratic differentials is given by:

$$
\begin{array}{lcl}
\phi_{1}(x)=\left(\frac{d x_{1}}{J^{1}(x)}\right)^{2} & \phi_{2}(x)=x_{1}\left(\frac{d x_{1}}{J^{1}(x)}\right)^{2} & \phi_{3}(x)=x_{2}\left(\frac{d x_{1}}{J^{1}(x)}\right)^{2}(6.7) \\
\phi_{4}(x)=x_{3}\left(\frac{d x_{1}}{J^{1}(x)}\right)^{2} & \phi_{5}(x)=\left(\frac{x_{1} d x_{1}}{J^{1}(x)}\right)^{2} & \phi_{6}(x)=\left(\frac{x_{2} d x_{1}}{J^{1}(x)}\right)^{2}(6.8) \\
\phi_{7}(x)=\left(\frac{x_{3} d x_{1}}{J^{1}(x)}\right)^{2} & \phi_{8}(x)=x_{1} x_{2}\left(\frac{d x_{1}}{J^{1}(x)}\right)^{2} & \phi_{9}(x)=x_{1} x_{3}\left(\frac{d x_{1}}{J^{1}(x)}\right)^{2}
\end{array}
$$

Clearly, a quadratic differential of the kind $\phi_{10}(x)=x_{2} x_{3}\left(\frac{d x_{1}}{J^{1}(x)}\right)^{2}$ would not be independent due to eq. (5.2). 
It is also easy to check that the following quadratic differential:

$$
K_{2}(x, y)=-\left(\frac{d x_{1}}{J^{1}(x)}\right)^{2}\left[\frac{x_{3} f\left(y_{1}, y_{2}, x_{3}\right)}{\left(x_{1}-y_{1}\right)\left(x_{3}-y_{3}\right)}+\frac{y_{2} f\left(x_{1}, y_{2}, x_{3}\right)}{\left(x_{1}-y_{1}\right)\left(x_{2}-y_{2}\right)}\right]
$$

has only a simple pole at the point $x_{i}=y_{i}, i=1,2,3$. In fact, $K_{2}(x, y)$ is obtained multiplying together the Weierstrass kernel (5.7) and the holomorphic differential $\omega_{1}(x)$ of eq. (5.26). The zeros of the latter cancel exactly the poles of $K(x, y)$. Indeed, since $J^{1}(x)=\frac{\partial f}{\partial x_{2}}-x_{1} \frac{\partial f}{\partial x_{3}}$ and $f$ is a polynomial of degree three in $x_{3}$, we have that $J^{1}(x) \sim-\frac{1}{x^{\prime 3}}$ at infinity in the three branches in which eq. (5.13) is satisfied.

At this point we are ready to write the correlation functions of the $b-c$ systems with $\lambda=2$ on $C_{4}$ :

$$
\begin{gathered}
\mathcal{G}_{2}(p ; q)= \\
\operatorname{det}\left|\begin{array}{cccccc}
K_{2}\left(x\left(p_{1}\right), y\left(q_{1}\right)\right) & \ldots & K_{2}\left(x\left(p_{1}\right), y\left(q_{1}\right)\right) & \phi_{1}\left(x\left(p_{1}\right)\right) & \ldots & \phi_{9}\left(x\left(p_{1}\right)\right) \\
\vdots & \ddots & \vdots & \vdots & \ddots & \vdots \\
K_{2}\left(x\left(p_{m}\right), y\left(q_{1}\right)\right) & \ldots & K_{2}\left(x\left(p_{m}\right), y\left(q_{n}\right)\right) & \phi_{1}\left(x\left(p_{m}\right)\right) & \ldots & \phi_{9}\left(x\left(p_{m}\right)\right)
\end{array}\right|
\end{gathered}
$$

Due to the properties of determinants, the right hand side of the above equation has the desired simple zeros whenever $x_{i}\left(p_{\alpha}\right)=x_{i}\left(p_{\alpha^{\prime}}\right), \alpha, \alpha^{\prime}=1, \ldots, m$ and $y_{i}\left(q_{\beta}\right)=y_{i}\left(q_{\beta^{\prime}}\right)$, $\beta, \beta^{\prime}=1, \ldots, n, i=1,2,3$. Moreover, all the poles of $\mathcal{G}_{2}(p ; q)$ are simple and occur at the points in which $x_{i}\left(p_{\alpha}\right)=y_{i}\left(q_{\beta}\right)$.

In principle, there could be also spurious poles due to the fact that $K_{2}(x, y)$ diverges when the variable $y$ becomes very large. However, from what it has been discussed in the previous Section, it is easy to realize that $K_{2}(x, y)$ has the following behavior in $y_{1}=\infty$ :

$$
K_{2}(x, y) \sim \sum_{i=1}^{4} \omega_{i}(x) \mathcal{A}_{i}(y)
$$


where the functions $\mathcal{A}_{i}$ have been defined in eq. (5.24) and diverge when $y_{1} \longrightarrow \infty$. Terms of the form (6.12) consist in a linear combination of quadratic differentials, which does not contribute in the determinant of eq. (6.11).

Let us now treat the case $\lambda=1$. On the Riemann surface $\Sigma_{4}$ the nonvanishing correlations functions are given by:

$$
\mathcal{G}_{1}(p, q)=\left\langle\prod_{\alpha=1}^{m} b\left(p_{\alpha}\right) \prod_{\beta=1}^{n} c\left(q_{\alpha}\right)\right\rangle
$$

where $m-n=g-1=3$. Again, the poles and zeros of the above correlator are determined by the physical properties of the $b-c$ fields. The divisors of $\mathcal{G}_{1}(p, q)$ are similar to those of $\mathcal{G}_{2}(p, q)$ with simple zeros whenever $p_{\alpha}=p_{\alpha^{\prime}}$ or $q_{\beta}=p_{\beta^{\prime}}$ and simple poles when $p_{\alpha}=q_{\beta}$, with $\alpha, \alpha^{\prime}=1, \ldots, m, \beta, \beta^{\prime}=1, \ldots, n$.

The explicit construction of $\mathcal{G}_{1}(p ; q)$ on the ramified covering $C_{4}$ goes as follows. First of all, we define the differential:

$$
\nu_{y(q) y\left(q^{\prime}\right)}(x(p))=K(x(p), y(q))-K\left(x(p), y\left(q^{\prime}\right)\right)
$$

This is a differential of the third kind in $x(p)$, with two simple poles in $x(p)=y(q)$ and $x(p)=y\left(q^{\prime}\right)$ and residues +1 and -1 respectively. The spurious poles of $K(x(p), y(q))$ in $x_{1}=\infty$ are canceled against the analogous poles of $K\left(x(p)-y\left(q^{\prime}\right)\right)$. At this point it is possible to write the expression of $\mathcal{G}_{1}(p ; q)$ :

$$
\begin{gathered}
\mathcal{G}_{1}(p ; q)= \\
\operatorname{det}\left|\begin{array}{cccccc}
\nu_{y\left(q_{1}\right) y\left(q_{n}\right)}\left(x\left(p_{1}\right)\right) & \ldots & \nu_{y\left(q_{n-1}\right) y\left(q_{n}\right)}\left(x\left(p_{1}\right)\right) & \omega_{1}\left(x\left(p_{1}\right)\right) & \ldots & \omega_{4}\left(x\left(p_{1}\right)\right) \\
\vdots & \ddots & \vdots & \vdots & \ddots & \vdots \\
\nu_{y\left(q_{1}\right) y\left(q_{n}\right)}\left(x\left(p_{m}\right)\right) & \ldots & \nu_{y\left(q_{n-1}\right) y\left(q_{n}\right)}\left(x\left(p_{m}\right)\right) & \omega_{1}\left(x\left(p_{m}\right)\right) & \ldots & \omega_{4}\left(x\left(p_{m}\right)\right)
\end{array}\right|
\end{gathered}
$$


As in the case $\lambda=2$, eq. (5.24) implies the absence of spurious poles in the $y$ variables in (6.15).

To conclude our list of conformal field theories which appear in bosonic string theory, we treat the scalar fields with action:

$$
S=\int_{\Sigma_{4}} d^{2} p \partial X \bar{\partial} X
$$

All the correlation functions of the scalar fields are obtained once the following correlator is known:

$$
G\left(p ; q, q^{\prime}\right)=\left\langle\partial_{p} X(p, \bar{p})\left[X(q, \bar{q})-X\left(q^{\prime}, \bar{q}^{\prime}\right)\right]\right\rangle d p
$$

It turns out that $G\left(p ; q, q^{\prime}\right)$ is a canonical differential of the third kind uniquely determined by the following properties:

a) $G\left(p ; q, q^{\prime}\right)$ has only two simple poles in $p=q$ and $p=q^{\prime}$ with residues +1 and -1 respectively.

b) The integral function $\int G\left(p ; q, q^{\prime}\right)$ has purely imaginary periods when transported around the $2 g=8$ non-trivial homology cycles of $\Sigma_{4}$.

On the algebraic curve $C_{4}$ the Green function (6.17) can be written as a vector field $G\left(x(p) ; y(q), y\left(q^{\prime}\right)\right)$, where $x(p)=x_{1}(p), x_{2}(p), x_{3}(p)$ etc. $G\left(x(p) ; y(q), y\left(q^{\prime}\right)\right)$ coincides to the third kind differential $\nu_{y(q) y\left(q^{\prime}\right)}(x(p))$ defined in eq. (6.14) up to a linear combination of holomorphic differentials, which is fixed by requirement $b$ ). In practice, since it is hard to deal with integrals over homology cycles in the case of algebraic curves, it is convenient to formulate this requirement in terms of surface integrals. Indeed, it is possible to show 
that $b)$ is satisfied if and only if $G\left(x(p) ; y(q), y\left(q^{\prime}\right)\right)$ fulfills the following Riemann bilinear identities:

$$
\int_{C_{4}} G\left(x(p) ; y(q), y\left(q^{\prime}\right)\right) \wedge \overline{\omega_{i}(x(p))}=0 \quad i=1, \ldots, 4
$$

The surface integrals over $C_{4}$ in $(6.18)$ can be interpreted as integrals in a three dimensional complex space concentrated in the solutions of eqs. (5.5) (see Appendix A). At this point we are able to write the Green function $G\left(x(p) ; y(q), y\left(q^{\prime}\right)\right)$ in terms of the third kind differential (6.14) and of the holomorphic differentials (5.25)-(5.26):

$$
G\left(x(p) ; y(q), y\left(q^{\prime}\right)\right)=\operatorname{det}\left|\begin{array}{cccc}
\nu_{y(q) y\left(q^{\prime}\right)}(x(p)) & \omega_{1}(x(p)) & \ldots & \omega_{4}(x(p)) \\
\int_{C_{4}} \nu_{y(q) y\left(q^{\prime}\right)} \wedge \overline{\omega_{1}} & \int_{C_{4}} \omega_{1} \wedge \overline{\omega_{1}} & \ldots & \int_{C_{4}} \omega_{1} \wedge \overline{\omega_{4}} \\
\vdots & \vdots & \ddots & \ldots \\
\int_{C_{4}} \nu_{y(q) y\left(q^{\prime}\right)} \wedge \overline{\omega_{4}} & \int_{C_{4}} \omega_{4} \wedge \overline{\omega_{1}} & \ldots & \int_{C_{4}} \omega_{4} \wedge \overline{\omega_{4}}
\end{array}\right|
$$

It is easy to see that the above differential of the third kind satisfies requirement $a$ ) and the relations (6.18), which are equivalent to $b)$.

\section{Conclusions}

In Section IV an analogue of the Weierstrass kernel has been constructed on non-plane algebraic curves associated to the vanishing of two polynomials $f$ and $g$. The freedom of adding linear combinations of differentials which do not change the behavior in $x=y$ has been exploited in order to get two different, but equivalent, versions of generalized Weierstrass kernels. The first version $K_{\text {sym }}(x, y)$, given in (4.1), is symmetric with respect to the variables $x, y$ and with respect to the exchange of $f$ with $g$. This is in agreement with the fact that neither the coordinates nor the functions $f$ and $g$ play a special role in the equations which define the curve. The alternative kernel $K(x, y)$ of eq. (4.7) has the 
advantage to have a more compact expression in comparison to $K_{\text {sym }}(x, y)$, but part of the symmetry under coordinate permutations is lost. If the branched cover of an algebraic curve is considered, the generalized Weierstrass kernel $K(x, y)$ has the simple form (4.8).

Furthermore, it has been verified that both kernels $K_{\text {sym }}(x, y)$ and $K(x, y)$ are third kind differentials with a simple pole in the desired point $x=y$ of the curve, in agreement with property (3.2), which characterizes the analogues of the Cauchy kernel on Riemann surfaces [3]. In Section IV it has also been proved that spurious singularities may only occur at the points in which one or more of the components of the coordinates $x$ or $y$ approach infinity. In the absence of a general algorithm to treat these spurious singularities like that developed in the case of plane curves in [22]-[23], the terms to be subtracted in order to get the desired correlation functions should be derived separately for any given polynomials $f$ and $g$. The example of a general non-hyperelliptic curve of genus four has been explicitly worked out and the amplitudes of bosonic string theory have been computed (see eqs. (6.11), (6.15) and (6.19)).

Finally, nothing has been said about non-hyperelliptic Riemann surfaces obtained from the intersection of $n-1$ hypersurfaces in $\mathbf{P}^{n}$ with $n>3$. However, it is clear from eqs. (4.1) and (4.7) what should be the structure of the generalized Weierstrass kernel on these curves. The Jacobians $J^{i}(x)$ should be replaced by analogous Jacobians containing derivatives of the $n-1$ polynomials $f_{1}, \ldots, f_{n-1}$ with respect to any possible $n-1-$ dimensional subsets of the coordinates. The numerators $N^{i}(x, y)$ will contain a sum of products of polynomials $f_{1} f_{2} \ldots f_{n-1}$, in which the dependence on the variables $x_{1}, \ldots, x_{n}$ and $y_{1}, \ldots, y_{n}$ is chosen in such a way that the spurious poles in the denominator given 
by the factor $\prod_{i=1}^{n}\left(x_{i}-y_{i}\right)$ are canceled, so that only the desired singularity in $x_{i}=y_{i}$, $i=1, \ldots, n$ remains.

\section{Acknowledgements}

This work has been completed during a visit at the Center for Theoretical Physics at MIT funded by a Senior CNR-NATO grant, which is gratefully acknowledged. I am indebted to R. Jackiw for the warm hospitality at CTP. I wish also to thank J. Sobczyk and W. Urbanik for fruitful discussions.

\section{Appendix A. Surface integrals over $C_{4}$}

In this Appendix we show that the surface integrals appearing in eqs. (6.18) and (6.19) can be expressed as integrals in a three dimensional complex space in the presence of Dirac $\delta$-functions which impose the constraints (5.5). We suppose here that $x \in \mathbf{C}^{3}$, but the result is valid also for a compact curve, in which case $\mathbf{C}^{3}$ has to be replaced by $\mathbf{P}_{1}^{3}$ and the non-flat metric of $\mathbf{P}_{1}$ should be taken into account.

Let us consider a surface integral of the kind:

$$
I=\int_{C_{4}} \rho
$$

where $\rho(x(p))$ is a $(1,1)$-form. In components $\rho(x(p))=\rho_{x_{1} \bar{x}_{1}}(x(p)) d x_{1} \wedge d \bar{x}_{1}$ and

$$
I=\int_{C_{4}} d^{2} x_{1}(p) \rho_{x_{1} \bar{x}_{1}}\left(x_{1}(p), x_{2}(p), x_{3}(p)\right)
$$


Since $C_{4}$ is a curve associated to the system of algebraic equations (5.5), it is possible to rewrite the integral (A.1) as follows:

$$
\int_{C_{4}} d^{2} x_{1}(p) \rho_{x_{1} \bar{x}_{1}}(x(p))=\int_{\mathbf{C}^{3}} d^{6} x\left|J\left(\begin{array}{cc}
f & g \\
x_{2} & x_{3}
\end{array}\right)\right|^{2} \delta^{(4)}(f, g) \rho_{x_{1} \bar{x}_{1}}(x(p))
$$

where $d^{6} x$ is the volume element in $\mathbf{C}^{3}$,

$$
J\left(\begin{array}{cc}
f & g \\
x_{2} & x_{3}
\end{array}\right)=\operatorname{det}\left|\begin{array}{ll}
\frac{\partial f}{\partial x_{2}} & \frac{\partial g}{\partial x_{2}} \\
\frac{\partial f}{\partial x_{3}} & \frac{\partial g}{\partial x_{3}}
\end{array}\right|
$$

and the Dirac $\delta$-function $\delta^{(4)}(f, g)$ has been defined using the formulas of [43]. After performing the change of variables $x_{2}, x_{3} \rightarrow f, g$, this distribution becomes an usual fourdimensional $\delta$-function:

$$
\delta^{(4)}(f, g)=\left(\frac{\partial_{f} \partial_{\bar{f}}+\partial_{g} \partial_{\bar{g}}}{2 \pi^{2}}\right) \frac{1}{|f|^{2}+|g|^{2}}
$$

Now let us apply to eq. (A.3) the inverse transformation which brings back to the old coordinates. This can be done using the relations:

$$
\partial_{f}=\frac{1}{g_{x_{2}} f_{x_{3}}-g_{x_{3}} f_{x_{2}}}\left(g_{x_{2}} \partial_{x_{3}}-g_{x_{3}} \partial_{x_{2}}\right) \quad \partial_{f}=\frac{1}{g_{x_{2}} f_{x_{3}}-g_{x_{3}} f_{x_{2}}}\left(f_{x_{3}} \partial_{x_{2}}-f_{x_{2}} \partial_{x_{3}}\right)
$$

Substituting the result in eq. (A.3), we obtain an explicit expression of $I$ in terms of three dimensional complex integrals:

$$
\begin{gathered}
\int_{C_{4}} d^{2} x_{1}(p) \rho_{x_{1} \bar{x}_{1}}(x(p)) \\
=\frac{1}{2 \pi^{2}} \int d^{6} x \rho_{x_{1} \bar{x}_{1}}(x(p))\left[\left|\left(g_{x_{2}} \partial_{x_{3}}-g_{x_{3}} \partial_{x_{2}}\right)\right|^{2}+\left|\left(f_{x_{3}} \partial_{x_{2}}-f_{x_{2}} \partial_{x_{3}}\right)\right|^{2}\right] \frac{1}{|f|^{2}+|g|^{2}}
\end{gathered}
$$




\section{References}

[1] H. Farkas and I. Kra, Riemann Surfaces, Springer Verlag, 1980.

[2] J. D. Fay, Theta Functions on Riemann Surfaces, Lecture Notes in Mathematical Physics no. 352, Springer Verlag, 1973.

[3] E.I. Zverovich, Russ. Math. Surv. 21 1971, 99.

[4] F. Enriques and O. Chisini, Lezioni sulla Teoria Geometrica delle Equazioni e delle Funzioni Algebriche, Zanichelli, Bologna (in italian).

[5] J. Harris and D. Eisenbud, Bull. Am. Math. Soc. 21 (2) (1989), 205; B. Dubrovin, Jour. Diff. Geom. Suppl. 4 (1998), 213; E. Ballico, C. Keem, Israel Jour. Math. 104 (1998), 355; C. Ciliberto, J. Harris, Commun. Algebra 27 (3) (1999), 2197.

[6] P. Griffiths and J. Harris, Principles of Algebraic Geometry, John Wiley \& Sons, New York 1978.

[7] Some Applications of hyperelliptic curves in perturbative string theory: R. Iengo and C. J. Zhu, Jour. High Energy Phys. 4 (2000), U651; R. Iengo and C.-J. Zhu, JHEP 9906 (1999), 011. D. Lebedev and A. Morozov, Nucl. Phys. B302 (1986), 163; E. Gava, R. Iengo and G. Sotkov, Phys. Lett. 207B (1988), 283; A. Yu. Morozov and A. Perelomov, Phys. Lett. 197B (1987), 115; D. Montano, Nucl. Phys. B297 (1988), 125; F. Ferrari, Fizika 21 (1989), 32; J. Sobczyk, Mod. Phys. Lett. A6 (1991), 1103; D. Montano, Nucl. Phys. B297 (1988), 125; E. Gava, R. Iengo and C. J. Zhu, Nucl. Phys. B323 (1989), 585; R. Jengo and C. J. Zhu, Phys. Lett. 212B (1988), 313; V. G. Knizhnik, Phys. Lett. 196B (1987), 473; D. J. Gross and P. F. Mende, Nucl. Phys. B303 (1988), 407. 
[8] Some applications of algebraic curves to various non-perturbative aspects of string theory and related problems: W. Lerche, On the Heterotic Theory Duality in EightDimensions, Proceedings of the TMR Summer School on Progress in String Theory and M-Theory (Cargese 99), Cargese, Corsica, France, 24 May - 5 Jun 1999, hepth/9910207; W. Lerche and S. Stieberger, Adv. Theor. Math. Phys. 2 (1998), 1105, hepth/9804176; W. Lerche, S. Stieberger and N. P. Warner, Quartic Gauge Couplings from K3 Geometry, Preprint CERN-TH/98-378, hep-th/9811228; G. Bertoldi, J. M. Isidro, M. Matone and P. Pasti, Phys. Lett. B484 (2000) 323 [hep-th/0003200]; G. Bertoldi, J. M. Isidro, M. Matone and P. Pasti, hep-th/0003131; M. Matone, Int. J. Mod. Phys. A10 (1995) 289 [hep-th/9306150]; N. Seiberg and E. Witten, Nucl. Phys. B430 (1994), 485; N. Seiberg and E. Witten, Nucl. Phys. B431 (1994), 484; J. M. Isidro, Journal Geom. Phys. 29 (1999), 334; E. Witten, Comm. Math. Phys. 113 (1988), 529.

[9] Some applications of algebraic curves in conformal field theories: Al. B. Zamolodchikov, Nucl. Phys. B285 (1987), 481; M. A. Bershadsky and A. O. Radul, Int. Jour. Mod. Phys. A2 (1987), 165; V. G. Knizhnik, Comm. Math. Phys. 112 (1987), 587; L. Borisov, M.B. Halpern and C. Schweigert, Int. Jour. Mod. Phys. A13 (1) (1998), 125; S. A. Apikian and C. J. Efthimiou, Int. Jour. Mod. Phys., A12 1997, 4291, hepth/9610051; S. A. Apikian and C. J. Efthimiou, Phys. Lett. 383B (1996), (397); C. J. Efthimiou and D. A. Spector, A Collection of Exercises in Two-Dimensional Physics, Part 1, Preprint CLNS-99-1612, hep-th/0003190; C. Crnkovic, G. M. Sotkov and M. Stanishkov, Phys. Lett. 220B (1989), 397; M. A. Bershadsky and A. Radul, Phys. Lett. 193B (1987), 21. 
[10] Some applications of algebraic curves to polymer physics: A. L. Kholodenko and T. A. Vilgis, Phys. Rep. 298 (1998), 251; S. Nechaev, Int. Jour. Mod. Phys. B4 (1990), 1809; Statistics of Knots and Entangled Random Walks, extended version of lectures presented at Les Houches 1998 Summer School on Topological Aspects of Low Dimensional Systems, July 7 - 31, 1998, cond-mat/9812205.

[11] Some other applications of algebraic curves: L. A. Takhtajan, Lett. Math. Phys. 52 (2000), 79; I. Bakas and K. Sfetsos, Nucl. Phys. B573 (2000), 768; S. M. Sergeev, Jour. Nonlinear. Math. Phy. 7 (2000), 57; B. Crespi, S. J. Chang, K. J. Shi, Jour. Math. Phys. 34 (6) (1993), 2257; S. Cordes, G. Moore and S. Rangoolam, Comm. Math. Phys. 185 (1997), 543; L. Dixon, D. Friedan, E. Martinec and S Shenker, Nucl. Phys. B282 (1987), 13; V. Marotta and A. Sciarrino, Mod. Phys. Lett. A13 (1998), 2863; V. Marotta, Nucl.Phys. B527 (1998), 717-737, hep-th/9702143.

[12] K. Weierstrass, Vorlesungen über di Theorie der Abelschen Transcendenten, Math. Werke. Vol. 4, Berlin 1902.

[13] D. Friedan, E. Martinec and S. Shenker, Nucl. Phys. B271 (1986), 93.

[14] E. Verlinde and H. Verlinde, Nucl. Phys. B288 (1987), 357; L. Alvarez-Gaumé, C. Gomez, P. Nelson, G. Sierra and C. Vafa, Nucl. Phys. B311 (1988), 333.

[15] M. Bershadsky and M. Radul, Comm. Math. Phys. 116 (1988), 689.

[16] F. Ferrari, Int. Jour. Mod. Phys. A5 (1990), 2799.

[17] F. Ferrari, Int. Jour. Mod. Phys. A7 (1992), 5131.

[18] M. A. Bershadsky and A. O. Radul, Int. Jour. Mod. Phys. A2 (1987), 165.

[19] H. Rauch, Comm. Pure Appl. Math. 12 (1959), 543. 
[20] A. A. Beilinson and Yu. I. Manin, Comm. Math. Phys. 107 (1986), 359.

[21] A. Nakayashiki, Publ. Res. Inst. Math. Sci. 34 (1998), 439; ibid. 33 (1997), 987.

[22] F. Ferrari and J. Sobczyk, Int. Jour. Mod. Phys. A11 (1996), 2213.

[23] F. Ferrari and J. Sobczyk, Journal Geom. Phys. 19 (1996), 287.

[24] F. Ferrari and J. T. Sobczyk, Jour. Math. Phys. 41 (9) (2000), 6444, hep-th/9909173.

[25] F. Ferrari and J. T. Sobczyk, Jour. Math. Phys. 39 (10) (1998), 5148.

[26] F. Ferrari, Lett. Math. Phys. 24 (1992), 165; F. Ferrari, Jour. Geom. Phys. 25 (1,2) (1998), 91; J. Sobczyk, Mod. Phys. Lett. A8 (1993), 1153.

[27] V. G. Knizhnik, Sov. Phys. Usp. 32(11) (1989) 945.

[28] I. M. Krichever and S. P. Novikov, Funk. Anal. Pril. 21 No.2 (1987), 46; 21 No.4 (1988), 47.

[29] R. Dick, Lett. Math. Phys. 18 (1989), 255; M. Schlichenmaier, Lett. Math. Phys. 19 (1990), 151.

[30] F. Ferrari, J. Sobczyk and W. Urbanik 36 (1995), 3216, hep-th/9310102.

[31] F. Ferrari, Comm. Math. Phys. 156 (1993), 179.

[32] M. Sato, T. Miwa and M. Jimbo. Holonomic quantum fields (Kyoto U.P. Kyoto), part I; 14 (1978) p. 223; II: 15 (1979) p. 201; III: 15 (1979) p. 577; IV: 15 (1979) p. 871; V; 16 (1980) p. 531.

[33] F. Ferrari, Int. Jour. Mod. Phys. A9 (3) (1994), 313.

[34] P. Griffiths, Principles of Algebraic Geometry, Wiley, New York 1978.

[35] M. Bonini and R. Jengo, Int. Jour. Mod. Phys. A3 (1988), 841; E. Date, M. Jimbo, M. Kashiwara and T. Miwa, In: Proc. of International Symposium on Nonlinear Integrable 
Systems, Kyoto 1981, M. Jimbo and T. Miwa (eds.), Singapore (1983); S. Saito, Phys. Rev. Lett. 36 (1987), 1819; L. Alvarez-Gaumé, C. Gomez and C. Reina, New Methods in String Theory, in: Superstrings '87, L. Alvarez-Gaumé (ed.), Singapore, World Scientific 1988; N. Ishibashi, Y. Matsuo and Y. Ooguri, Mod. Phys. Lett. A2 (1987), 119; N. Kawamoto, Y. Namikawa, A. Tsuchiya and Y. Yamada, Comm. Math. Phys. 116 (1988), 247.

[36] L. Bonora, A. Lugo, M. Matone and J. Russo, Comm. Math. Phys. 123 (1989), 329.

[37] L. Bonora, M. Matone, F. Toppan and K. Wu, Phys. Lett. 224B (1989), 115; Nucl. Phys. B334 (1990), 717; L. Bonora and F. Toppan, Rev. Math. Phys. 4 (1992), 429.

[38] C. Vafa, Phys. Lett. 190B (1987), 47.

[39] A. K. Raina, Comm. Math. Phys. 122 (1989), 625; ibid. 140 (1991), 373; Lett. Math. Phys. 19 (1990), 1; Expositiones Mathematicae 8 (1990), 227; Helvetica Physica Acta 63 (1990), 694.

[40] P. di Vecchia, Phys. Lett. B248 (1990), 329; P. Di Vecchia, F. Pezzella, M. Frau, K. Hornfleck, A. Lerda and A. Sciuto, Nucl. Phys. B332 (1989), 317; ibid. B333 (1990), 635; A. Clarizia and F. Pezzella, Nucl. Phys. B298 (1988), 636; G. Cristofano, R. Musto, F. Nicodemi and R. Pettorino, Phys. Lett. 217B (1989), 59.

[41] A. Lugo and J. Russo, Nucl. Phys. B322 (1989), 210; J.Russo, Phys. Lett. 220B (1989), 104.

[42] A. M. Semikhatov, Phys. Lett. B212 (1988), 357; O. Lechtenfeld, Phys. Lett B232 (1989) 193; U. Carow-Watamura and S. Watamura, Nucl. Phys. B288 (1987), 500; Nucl. Phys. B301 (1988), 132; Nucl. Phys. B302 (1988), 149; Nucl. Phys. B308 
(1988), 143; U. Carow-Watamura, Z. F. Ezawa, K. Harada, A. Tezuka and S. Watamura, Phys. Lett. B227 (1989), 73.

[43] I. M. Gel'fand and G. E. Shilov, Generalized functions, Vol. I, Academic Press, New York and London, 1964. 